\title{
A Generative Method for Textured Motion: Analysis and Synthesis
}

\author{
Yizhou Wang and Song-Chun Zhu \\ Dept. of Comp. and Info. Sci., Ohio State Univ., Columbus, OH 43210, USA \\ \{wangyiz, szhu\}@cis.ohio-state.edu
}

\begin{abstract}
Natural scenes contain rich stochastic motion patterns which are characterized by the movement of a large number of small elements, such as falling snow, raining, flying birds, firework and waterfall. In this paper, we call these motion patterns textured motion and present a generative method that combines statistical models and algorithms from both texture and motion analysis. The generative method includes the following three aspects. 1). Photometrically, an image is represented as a superposition of linear bases in atomic decomposition using an overcomplete dictionary, such as Gabor or Laplacian. Such base representation is known to be generic for natural images, and it is low dimensional as the number of bases is often 100 times smaller than the number of pixels. 2). Geometrically, each moving element (called moveton), such as the individual snowflake and bird, is represented by a deformable template which is a group of several spatially adjacent bases. Such templates are learned through clustering. 3). Dynamically, the movetons are tracked through the image sequence by a stochastic algorithm maximizing a posterior probability. A classic second order Markov chain model is adopted for the motion dynamics. The sources and sinks of the movetons are modeled by birth and death maps. We adopt an EM-like stochastic gradient algorithm for inference of the hidden variables: bases, movetons, birth/death maps, parameters of the dynamics. The learned models are also verified through synthesizing random textured motion sequences which bear similar visual appearance with the observed sequences.
\end{abstract}

\section{Introduction: Objectives and Previous Work}

Natural scenes contain rich stochastic motion patterns which are characterized by the movement of a large number of small deformable elements (or particles). For example, raining, snowing, bird flock, moving crowd, firework, waterfalls, and so on. The analysis and synthesis of such motion patterns, called textured motion in this paper, are important for a variety of applications in both vision and graphics, and stimulate growing interest of the two communities.

Graphics methods. In graphics, the objective is to render textured motion in video or cartoon animation, and the quality of the rendered motion is usually measured by three basic criteria. 
1. It should be realistic. This motivates work for modeling and learning the photometric and dynamic properties from real video due to the complexity of textured motion. Usually, data driven statistical modeling is often more appropriate than physically-based modeling.

2. It should be stylish. This is required for applications in non-photo realistic rendering (NPR), for example, rendering a waterfall in a cartoon movie. It is desirable to separate the dynamics of motion from its photometric appearances, so that the video appears symbolic but with realistic motion.

3. It should be controllable. For a better blending of the motion with other $3 \mathrm{D}$ objects in a scene, one should increase the degree of freedoms in maneuvering the motion. For example, it is desirable to control the sources and sinks where the motion elements appear and disappear, to control the individual moving elements, to change its motion direction etc.

In the graphics literature, both physically-based and data driven models are reported. The former includes the work which create animations of fire and gaseous phenomena with particles $[12,5]$. The latter includes the 1$)$. video texture[14] which finds smooth transition points in a video sequence from which the video could be replayed with minimum artifacts; 2 ). 3D volume texture[18] which generates motion through non-parametric sampling from an observed video motivated by recent work on texture synthesis. Though the statistical models of the video texture or $3 \mathrm{D}$ volume texture can render some realistic animations, such models do not model the dynamic and geometric properties of the moving elements.

Vision methods. In computer vision, the analysis of textured motion has applications for video analysis, such as motion segmentation, annotation, recognition and retrieval, detecting abnormal motion in a crowd, and so on. Needless to say that a good vision model of textured motion is useful for animation in graphics as mentioned above. For such applications, a vision model should satisfy the following properties.

1. It should be sufficient and general. It is not enough to just render a synthesized sequence that looks like the original as the video texture do, the model should also be able to capture the variability and therefore can be generalized to new data.

2. It should be parsimonious and low dimensional for computation. This requests the model capture the semantics of the motion. This also requests the modeling of photometric, geometric, and dynamic aspects of the motion - consistent with the graphics criteria.

In the vision literature, as these motion patterns lie in the domains of both motion analysis and texture modeling, statistical models are proposed from both directions with a trend of merging the two. In the following, we briefly review these work to set the background of our method.

Early vision work on textured motion was done by (Szummer and Picard, 1996)[17] who adopt a spatial-temporal auto-regression (STAR) model from 
(Cliff and Ord, 1976)[4]. Let $\mathbf{I}(x, y, t)$ be the intensity of a pixel $(x, y)$ at time $t$, a STAR model assumes that $\mathbf{I}(x, y, t)$ is a regression of its neighboring pixels

$$
\mathbf{I}(x, y, t)=\sum_{i=1}^{p} a_{i} \mathbf{I}\left(x+\delta x_{i}, y+\delta y_{i}, t+\delta t_{i}\right)+N\left(0, \sigma^{2}\right),
$$

where $\left(\delta x_{i}, \delta y_{i}, \delta t_{i}\right)$ is the displacement of a neighboring pixel in space and time, and $a_{i}, i=1, \ldots, p$ are parameters to be fit. A linear (or partial) order is imposed so that $\mathbf{I}(x, y, t)$ only depends on pixels at previous frames $\delta t_{i}<0, \forall i$ for fast synthesis. Such model can be considered as an extension from a causal Gaussian Markov random field model (GMRF) used in texture modeling by adding the time dimension. Along the line of texture modeling, Bar-Joseph et.al.[1] extended the work by Heeger and Bergen (1995) and others[19] to multi-resolution analysis in a tree structured representation, in a similar spirit to (Wei and Lovoy, 2000).

Although these algorithms can show synthesis of good motion, we argue that the concept of treating a motion pattern as a solid texture is perhaps not appropriate. Because textures are physically the status of systems with massive elements at thermodynamic equilibrium characterized by maximum entropy distributions[19]. However, this assumption is not observed in textured motions, for example, fire or gaseous turbulence, which are clearly not at equilibrium.

The recent work (Soatto, Doretto, and Wu, 2001)[15] engages the motion dynamics explicitly. By a SVD analysis, Soatto et al. represent an image $\mathbf{I}(t)$ by a small number of principal components. The projections of $\mathbf{I}(t)$ on these components, denoted by $x(t)$, is modeled by a Markov model,

$$
x(t+1)=A x(t)+B v(t), \quad \mathbf{I}(t)=C x(t)+n(t),
$$

where $v(t)$ is the noise driving the motion and $n(t)$ is the image noise for the reconstruction residues. The parameters $A, B, C$ are learned by maximum likelihood estimation (MLE). This model can generate impressive synthesis for a variety of motion patterns and can also be used for recognition[13].

Being considered as an extension the work [15], Fitzgibbon considered not only the stochastic part for textured motion, but also the parametric component introduced by the camera motion [16]. In [16], the images are also represented by the principal components with peroidc coefficients, and the Auto-Regression (AR) model is used to handel stochastic textured motion. The parametric component for camera motion is governed by projective geometry model. The objective of the method is to both efficiently fit the AR model and correctly register the image sequence.

Our method. In this paper, we present a generative method for the analysis and synthesis of textured motion, motivated by the vision and graphics criteria discussed above. Our model includes the following three aspects.

1. Photometrically, an image is represented as a superposition of linear bases in atomic decomposition using an over-complete dictionary, such as Gabor or Laplacian. Such base representation is known to be generic for natural 
images, and it is low dimensional as the number of bases is often 100 times smaller than the number of pixels.

2. Geometrically, each moving element (called moveton), such as the individual snowflake, bird, is represented by a template which is a group of several spatially adjacent bases. Such templates are deformable to account for the variabilities of the elements and are learned through clustering.

3. Dynamically, the movetons are tracked through the image sequence by a stochastic algorithm maximizing a posterior probability. A classic Markov chain model is adopted for the motion dynamics, as in[15]. The sources and sinks of the movetons are modeled by birth and death maps.

We adopt an EM-like stochastic gradient algorithm for inference of the hidden variables: bases, movetons, birth/death maps, parameters of the dynamics.

\section{A Generative Model for Images and Video}

To fix notation, let $\mathbf{I}[0, \tau]$ denote an image sequence on a $2 \mathrm{D}$ lattice $\Lambda=\{(x, y)$ : $0 \leq x, y \leq L\}$ in a discretized time interval $[0, \tau]=\{0,1,2, \ldots, \tau\}$. For $(x, y) \in A$ and $t \in[0, \tau], \mathbf{I}(x, y, t)$ denotes the pixel intensity, and $\mathbf{I}(t) \in \mathbf{I}[0, \tau]$ is a single image frame.

\subsection{Image Representation: From Pixels to Bases}

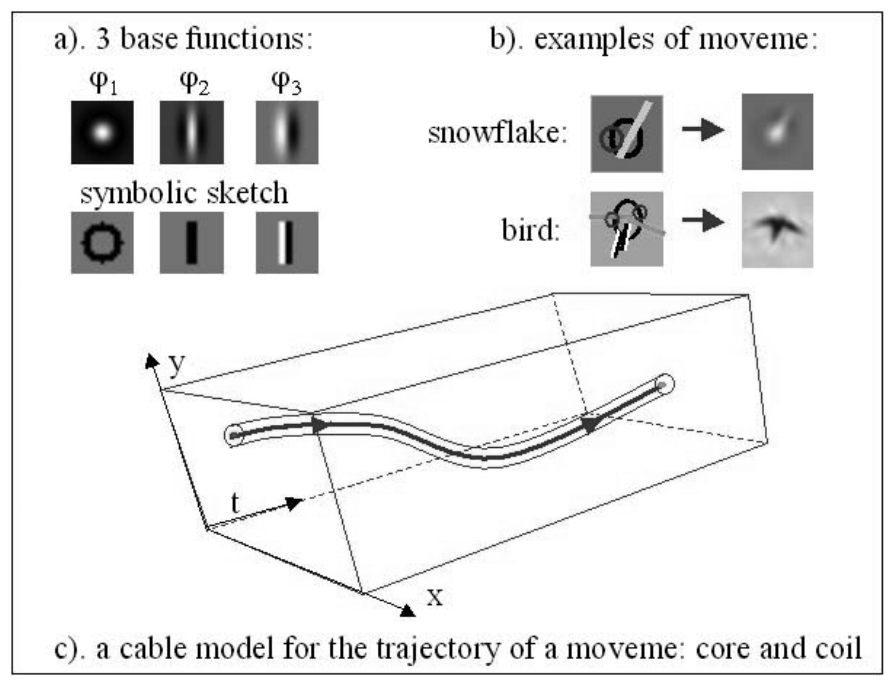

Fig. 1. A "cable model" for movetons.

In this section, we study the representation of a single image frame $\mathbf{I} \in \mathbf{I}[0, \tau]$. For clarity, we remove the time index. We represent an image as a superposition 
of a small number of image bases, in a scheme which is often called atomic decomposition in wavelets and image coding $[9,10,3]$.

$$
\mathbf{I}=\sum_{j=1}^{N} \alpha_{j} \mathbf{b}_{j}+\mathbf{n}, \quad \mathbf{b} \in \Delta .
$$

In equation (3), $\mathbf{b}_{j}$ is an image base from a dictionary $\Delta, \alpha_{j}$ is its coefficient, and $\mathbf{n}$ is a noise process for the residues. The dictionary includes all bases which are transformed versions of three base functions (mother wavelets) $\psi_{\ell}, \ell=1,2,3$,

$$
\Delta=\left\{T_{x, y, \theta, \sigma} \circ \psi_{\ell}:(x, y) \in \Lambda, \theta \in[0,2 \pi), \sigma \in\left[\sigma_{\min }, \sigma_{\max }\right], \ell=1,2,3\right\}
$$

$T_{x, y, \theta, \sigma}$ denotes a transform with $(x, y, \theta, \sigma)$ for translation, rotation, and scaling respectively.

We denote the set of base functions by $\Psi=\left\{\psi_{\ell}, \ell=1,2,3\right\}$. We choose the Laplacian of Gaussian (LoG), Gabor cosine (Gcos), and Gabor sine (Gsin) shown in Figure 1.a. These base functions represent blobs, bars and step edges respectively (see the symbolic sketches in Figure 1.a). We choose 8 scales, and 12 orientations.

Thus we transform an image $\mathbf{I}$ into a base representation, called a base map.

$$
\mathbf{B}=\left(\mathbf{b}_{j}=\left(\alpha_{j}, \ell_{j}, x_{j}, y_{j}, \theta_{j}, \sigma_{j}\right): j=1,2, \ldots, N\right) .
$$

As $\Delta$ is over-complete, we should discuss how $\mathbf{B}$ is inferred from $\mathbf{I}$ later. We choose the base representation for two reasons.

1. Low dimensionality. The number of bases is usually 100 -fold smaller than the number of pixels. Figure 2 shows a snowing sequence, each frame can be approximated by $N \approx 100$ bases (see Figure $2 . \mathrm{b}$ ). When $N$ increases to 800 bases, the reconstructed images in Figure 2.c) are of very high precision. This also introduces a coarse-to-fine strategy for computation.

2. Generality. It is well known that the LoG and Gabor bases are generic representations for the ensemble of natural images[11], and are also fundamental to human visual perception.

\subsection{Image Representation: From Bases to Movetons}

In natural image sequences, the image bases often form spatially coherent groups. This is most evident in sequences where the moving elements (or "movetons") are identifiable, such as the individual snow flakes, and flying birds. Figure 1.b shows two examples. A snow flake is a sum of three bases: $2 \psi_{1}$ 's and $1 \psi_{2}$ at various scales and space displacements. A bird consists of 7 bases: $3 \psi_{1}$ 's, $2 \psi_{2}$ 's $2 \psi_{3}$ 's. The number of bases, and their relative positions and coefficients may vary between the movetons. By defining a distance between the movetons, one can cluster the movetons into a small number of deformable templates.

$$
\Phi=\left\{\phi_{\ell}(\beta): \ell=1,2, \ldots, n\right\}
$$



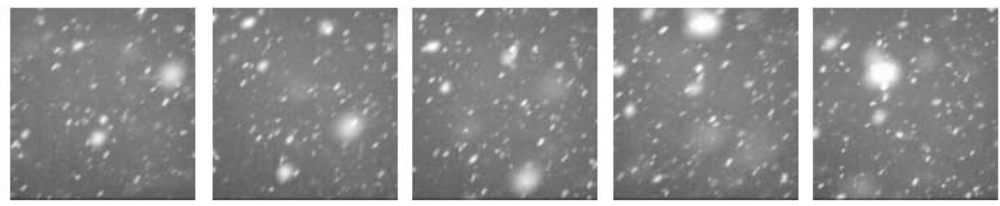

a). observed sequence
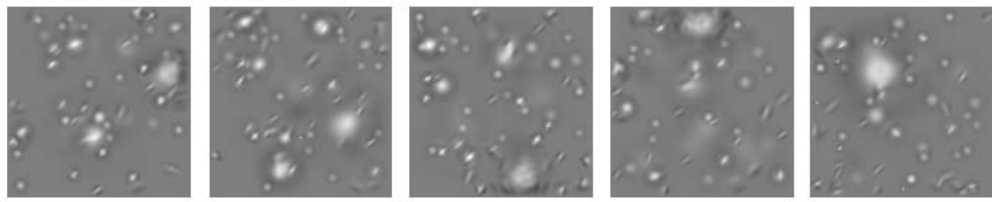

b). reconstructed sequence by $\sim \mathbf{1 0 0}$ bases per frame
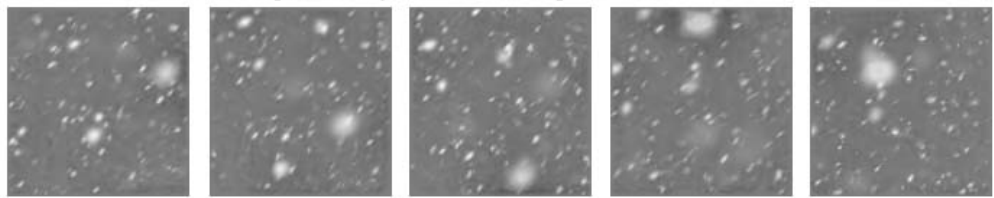

c). reconstructed sequence by $\sim \mathbf{8 0 0}$ bases/frame
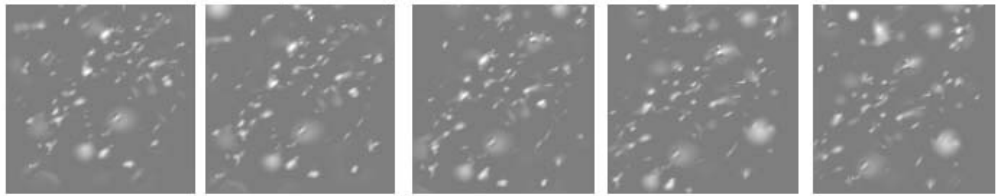

d). synthesized sequence by random sampling

Fig. 2. Example of a snowing sequence. (see snow_obs.avi and snow_syn.avi for movies)

with $\ell$ indexing the moveton types and $\beta$ being the parameters for relative deformations of the bases within a moveton. Thus we obtain a dictionary of movetons with some transformations,

$$
\Pi=\left\{T_{x, y, \theta, \sigma} \circ \phi_{\ell}:(x, y) \in \Lambda, \theta \in[0,2 \pi), \sigma \in\left[\sigma_{\min }, \sigma_{\max }\right], \ell\right\} .
$$

In practice, not all bases are necessarily grouped into movetons. We call the ungrouped ones free bases, which are treated as degenerated movetons, i.e. each moveton has one base, for clarity of notation. For the $N$ bases in the base map $\mathbf{B}$, suppose we group them into $J$ movetons, then we arrive at a more meaningful representation of the image, with dimensions further reduced than $\mathbf{B}$.

$$
\mathbf{M}=\left(\pi_{j}=\left(\ell_{j}, x_{j}, y_{j}, \theta_{j}, \sigma_{j}, \beta_{j}\right), j=1,2, \ldots, J\right), \quad J \ll N .
$$

Each moveton $\pi_{j}$ is represented by $1 \leq \ell_{j} \leq n$ for the type of the deformable template, $x_{j}, y_{j}, \theta_{j}, \sigma_{j}$ for the position, orientation, and scale of the overall moveton, and $\beta$ for the deformable within the moveton.

During the computation, we should learn the deformable templates $\Phi_{\ell}$ and compute the movetons and free bases $\mathbf{M}$ from images. For example, Figure 3.a displays the symbolic sketches for a set of typical deformable templates of the 


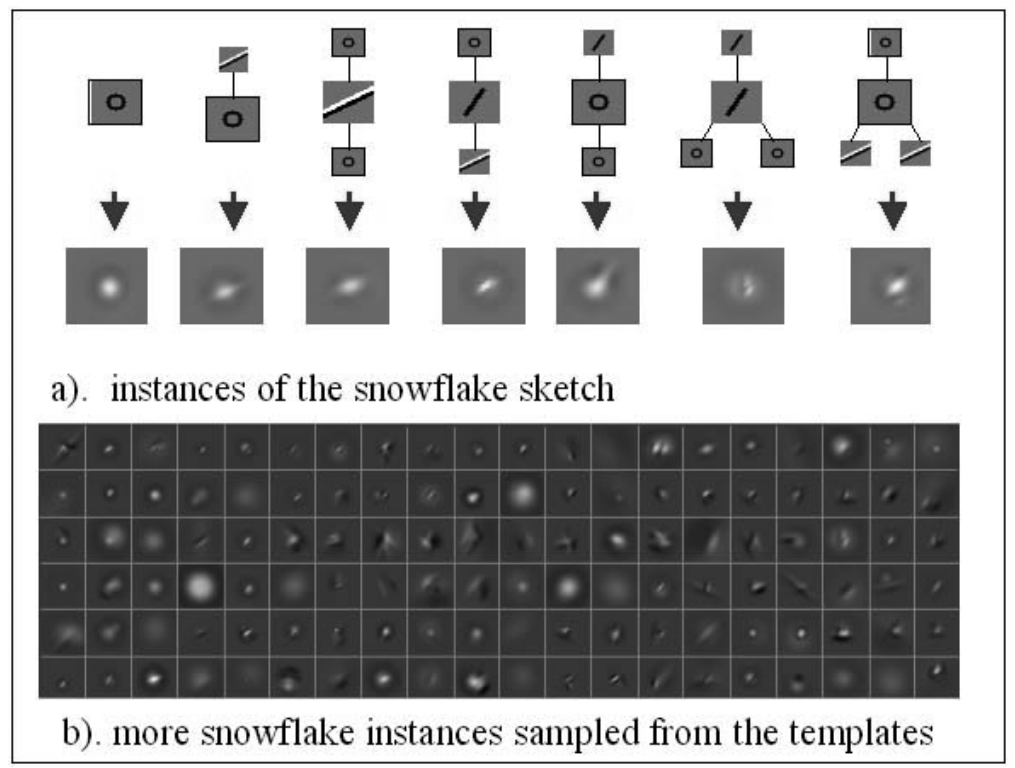

Fig. 3. The computed motion elements: snow flakes and random examples.

snowing sequence shown in Figure 2. Figure 3.b shows 120 random movetons sampled from the moveton dictionary $\Pi$. Each moveton is a snow flake. This sample shows the variety and generality of the deformable models learned with bases.

To summarize, we have a following generative model for an image $\mathbf{I}$, with dimensions reduced sequentially,

$$
\mathbf{M} \stackrel{\Phi}{\rightarrow} \mathbf{B} \stackrel{\Psi}{\longrightarrow} \mathbf{I}
$$

\subsection{Motion Representation: Dynamics, Sources, Sinks, and State Transition}

Now we turn to the image sequence $\mathbf{I}[0, \tau]$. As shown in Figure 1.c, a moveton $\pi$ can be traced over a certain time interval $\left[t_{b}, t_{e}\right]$ and thus its trajectory is what we call a "cable". Typically in a moveton template, one base has relatively large coefficient and scale, such as the main body of the bird or snow flake, and its trajectory forms the core of the cable. The core base is surrounded by a number of minor bases which account for the deformations. Due to self-rotation, the trajectories of these minor bases form the coil surrounding the cable core. In a coarse-to-fine computation, we can compute the trajectories of the cores first, and then add the coils sequentially. Thus we denote a cable by

$$
C\left[t^{b}, t^{e}\right]=\left(\pi\left(t^{b}\right), \pi\left(t^{b}+1\right), \ldots, \pi\left(t^{e}\right)\right) .
$$


In practice, the core of a moveton is relatively consistent through its life span, and the number of coil bases may change over time, due to self-occlusion etc. Since these bases are often minor, we assume the number of coil bases are fixed in a cable for simplicity.

We adopt a classic 2nd order Markov model which is sufficient for the dynamics of a moveton $C\left[t^{b}, t^{e}\right]$. In other words we fit the trajectory (the cable) $C\left[t^{b}, t^{e}\right]$ by regression. Such models are extensively used in tracking $[8]$.

$$
\begin{aligned}
\pi(t)= & A \pi(t-1)+B \cdot \pi(t-2)+C+D N\left(0, \sigma_{0}^{2}\right) \quad t \in\left[t^{b}+2, t^{e}\right] \\
& \pi\left(t^{b}+1\right)=A^{\prime} \pi\left(t^{b}\right)+C^{\prime}+D \omega \\
& \left(\pi\left(t^{b}\right), t^{b}\right) \sim P_{B}(\pi, \lambda), \quad\left(\pi\left(t^{e}\right), t^{e}-t^{b}\right) \sim P_{D}(\pi, \lambda) .
\end{aligned}
$$

One can simplify the equation in a canonical form expressed in equation (2). $\pi(t)$ is a vector representing a number of bases including both the photometric (by base coefficients) and geometric information. The matrices $A, B, C, D, A^{\prime}, C^{\prime}$ capture the change of image appearances and the motion of the movetons, and these matrices are usually diagonal. Since the motion patterns we are studying is textured motion, we assume that those movetons have similar dynamics. That means those trajectories share the same $A, B, C, D, A^{\prime}, C^{\prime}$.

The first moveton $\pi\left(t^{b}\right)$ and its timing $t^{b}$ follows a probability $P_{B}(\pi, \lambda)$ which we call the birth map for movetons. $P_{B}$ specifies the "sources" of the movetons where the movetons are often originated. Similarly, the end of the trajectory $\pi\left(t^{e}\right)$ and its life span $t^{e}-t^{b}$ are governed by a death map $P_{D}(\pi, \lambda) . P_{D}$ reveals the "sinks" in a lattice. $\pi$ is a long vector, $P_{B}$ and $P_{D}$ are high dimensional. Although other attributes in $\pi$ can be modeled if necessary, we are most interested in the location $(x, y)$.

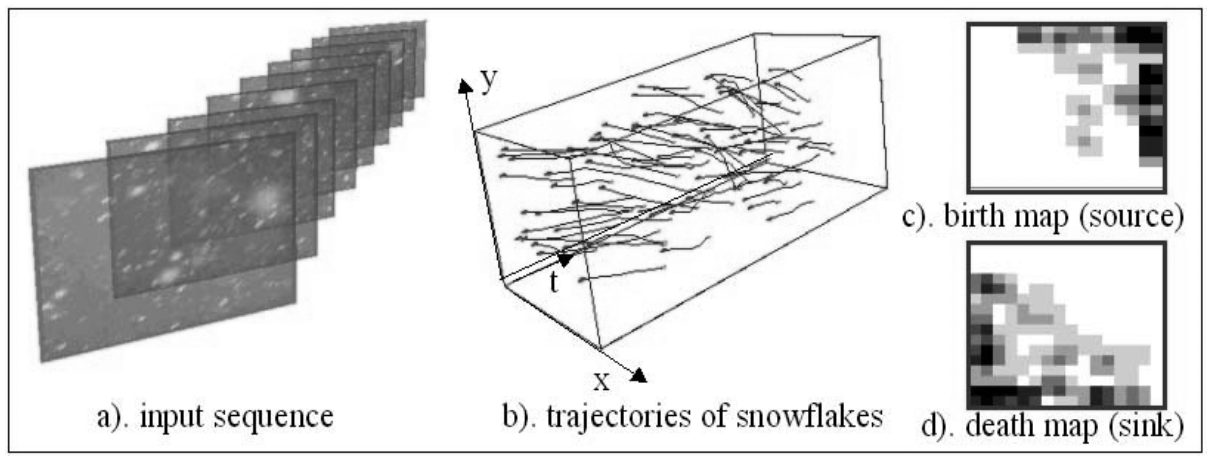

Fig. 4. The computed trajectories of snow flakes and the source and sink maps.

For example, Figure 4 displays the computed trajectories (4.b), birth (source) map (4.c), and death (sink) map (4.d) of the snowing sequence shown in Figure 2. The dark locations at the death/birth maps indicate high probabilities. Thus 
the algorithm "understands" that the snow flakes enter mostly from the upperright corner and disappear around the lower-left corner. We sum over the other variables at each $(x, y)$.

During the learning process, suppose we have computed $K$ cables from a sequence $\mathbf{I}[0, \tau], C_{i}\left[t_{i}^{b}, t_{i}^{e}\right], i=1,2, \ldots, K$, we represent $P_{B}$ and $P_{D}$ in a nonparametric form,

$P_{B}(\pi, \lambda)=\frac{1}{K} \sum_{i=1}^{K} \delta\left(\pi-\pi_{i}\left(t_{i}^{b}\right), \lambda-t_{i}^{b}\right), \quad P_{D}(\pi, \lambda)=\frac{1}{K} \sum_{i=1}^{K} \delta\left(\pi-\pi_{i}\left(t_{i}^{b}\right), \lambda-\left(t_{i}^{e}-t_{i}^{b}\right)\right)$

where $\delta()$ is a Parzen window centered at 0 . Then we can project $P_{B}$ and $P_{D}$ to the $(x, y)$ dimensions as marginal probabilities.

In practice, the death and birth of movetons may be synchronized. For example, in the firework scene shown in Figure 10, a large number of movetons can come and go together. This requests the $P_{B}$ and $P_{D}$ be joint probabilities for a large number of movetons.
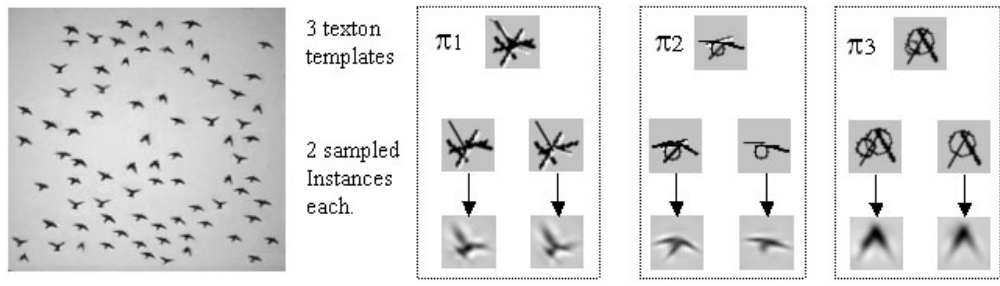

Fig. 5. Three transition states while birds flying.

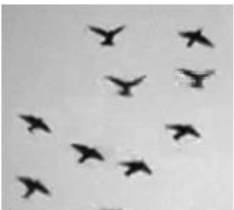

a

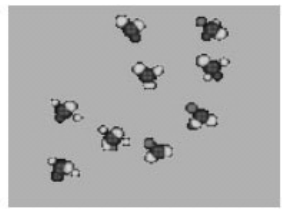

b

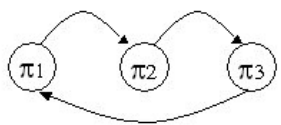

C

Fig. 6. 3D graphic model of flying birds and their flying states transition.

Furthermore, sometimes when the movetons are non-rigid objects or articulated objects, we may observe certain repeating states in their movements, for example, the birds flapping their wings while flying. Thus we also need to model the state transition of those movetons. As the result, we extend the motion dynamics model with more states. Figure 5 shows the clustered three states $(\pi 1$, $\pi 2, \pi 3)$ of the poses when birds flying. And Figure 6 displays the $3 \mathrm{D}$ graphic 
model for the birds and their flying states transition. During the synthesis of birds fly, once we determine the birds' flying pathes, we can make those birds flapping their wings by sampling the transition states from the model.

To summarize, we denote all parameters in the motion equation above by,

$$
\Gamma=\left(A, B, C, D, B, A^{\prime}, C^{\prime}, P_{B}, P_{D}, T\left(\pi_{j}\right)\right)
$$

\section{Problem Formulation}

Given an observed image sequence $\mathbf{I}^{\text {bbs }}[0, \tau]$ as training data, we want to achieve two objectives.

1. Make inference about all the hidden (latent) variable which are represented by an unknown of $K$ cables,

$$
W[0, \tau]=\left(K,\left\{\left(t_{i}^{b}, t_{i}^{e}, C_{i}\right):\left[t_{i}^{b}, t_{i}^{e}\right] \subset[0, \tau], i=1,2, \ldots, K\right\}\right) .
$$

2. Compute the optimal fit for all parameters in the generative model $\Theta=$ $(\Phi, \Gamma)$, with $\Phi$ being the set of deformable templates for the movements, and $\Gamma$ governing the birth, death, and motion of the movetons.

The formulation is standard in statistics for learning a model with latent variables (missing data), that is, the maximum likelihood estimate (MLE),

$$
\Theta^{*}=\left(\Phi^{*}, \Gamma^{*}\right)=\arg \max \log p\left(\mathbf{I}^{\text {obs }}[0, \tau] ; \Theta\right) .
$$

The likelihood is computed from the generative model with latent variables integrated (summed) out, For clarity of notation, we assume $W$ are continuous variables.

$$
p\left(\mathbf{I}^{\mathrm{obs}}[0, \tau] ; \Theta\right)=\int p\left(\mathbf{I}^{\mathrm{obs}}[0, \tau] \mid W[0, \tau] ; \Phi\right) p(W[0, \tau] ; \Gamma) d W .
$$

Let $\mathbf{B}(t)=\left\{\mathbf{b}_{t, j}, j=1,2, \ldots, N(t)\right\}$ be the collection of all bases in the $K$ movetons (cables) at time $t$, then we can re-express $W[0, \tau]$ as $(\mathbf{B}(0), \ldots, \mathbf{B}(\tau))$, by equation $(3), p(\mathbf{I}[0, \tau] \mid W[0, \tau] ; \Phi)$ is the product Gaussians,

$$
p\left(\mathbf{I}^{\mathrm{obs}}[0, \tau] \mid W[0, \tau] ; \Phi\right)=\prod_{t=0}^{\tau} G\left(\mathbf{I}^{\mathrm{obs}}(t)-\sum_{j=1}^{N(t)} \alpha_{t, j} \mathbf{b}_{t, j} ; \sigma_{o}^{2}\right),
$$

as we assume iid Gaussian noise $G\left(0, \sigma_{o}^{2}\right)$ for $\mathbf{n}$. sians,

Following the motion representation, $p(W[0, \tau] ; \Gamma)$ is also a product of Gaus-

$$
\begin{aligned}
p(W[0, \tau] ; \Gamma) & =\prod_{i=1}^{K} P_{B}\left(\pi\left(t_{i}^{b}\right), t^{b}\right) P_{D}\left(\pi\left(t_{i}^{e}\right), t_{i}^{e}\right) p\left(\pi\left(t_{i}^{b}+1\right) \mid \pi\left(t_{i}^{b}\right) ; A^{\prime}, C^{\prime}, D\right) \\
& \times \prod_{t=t_{i}^{b}+2}^{t_{i}^{e}} p(\pi(t) \mid \pi(t-1), \pi(t-2) ; A, B, C, D) .
\end{aligned}
$$




\section{Computation: Learning and Inference}

To solve the MLE in eqn. (6), we set $\frac{\partial \log p\left(\mathbf{I}^{\text {obs }} ; \Theta\right)}{\partial \Theta}=0$. This leads to

$$
\int\left[\frac{\partial \log p\left(\mathbf{I}^{\mathrm{obs}} \mid W ; \Phi\right)}{\partial \Phi}+\frac{\partial \log p(W ; \Gamma)}{\partial \Gamma}\right] p(W \mid \mathbf{I} ; \Theta) d W=0
$$

Instead of using the classic EM algorithm, we adopt the stochastic gradient algorithm[6] which is capable of being global optimal $\Theta$. It iterates three steps with $s$ indexing steps.

Step 1. Sampling $W^{\operatorname{syn}}[0, \tau] \sim p\left(W \mid \mathbf{I}^{\text {obs }} ; \boldsymbol{\beta}\right)$. This includes computing the bases, grouping bases into movetons, and tracking the movetons. The computation is realized by a data driven Markov chain Monte Carlo techniques, including the following reversible dynamics.

1). The death or birth of a motion trajectory $\Gamma$ of length one.

2). Extending or shrinking a trajectory.

3). Mutating two nearby trajectories at a certain base.

4). Diffusing the coefficient, location, orientation, scale of a base in a trajectory (Inferring $\mathbf{B}$ ).

Step 2. Updating the motion dynamics parameters $\Gamma$ by regression,

$$
\Gamma(s+1)=(1-\rho) \Gamma(s)+\rho \frac{\partial \log p\left(W^{\mathrm{syn}}[0, \tau] ; \Gamma\right)}{\partial \Gamma} .
$$

Step 3. Updating the moveton parameters $\Gamma$ by clustering and grouping,

$$
\Phi(s+1)=(1-\rho) \Phi(s)+\rho \frac{\partial \log p\left(\mathbf{I}^{\mathrm{obs}} \mid W^{\mathrm{syn}} ; \Phi\right)}{\partial \Phi} .
$$

Finally, the birth, death maps, $P_{B}$ and $P_{D}$, are updated by counting the the head and tail of each cable at their locations in the frames.

The algorithm is initialized by a stochastic version of match pursuit[9] for the base maps which is often very effective. We adopt a coarse-to-fine scheme and track the core bases whose coefficients and scales are higher than a threshold, and learn the motion dynamics $\Gamma$. Then we lower the threshold to add the coil bases quickly following the learned trajectory.

Our method for tracking movetons is similar to the condensation algorithm[8], while is distinguished from it in two main aspects. Firstly, we have a full generative model of image rather than the tracking model whose likelihood can only be evaluated relatively. Secondly, we are optimizing the whole trajectories and thus will trace back in time during the computation, which means we don't have to remember a huge samples for each movetons. This, in combination with the generative model, saves large amount of time and memory.

For a typical sequence of 30 frames, the learning takes about 10-20 minutes in a Pentium IV PC, and the synthesis of sequence can be done in nearly real-time. 

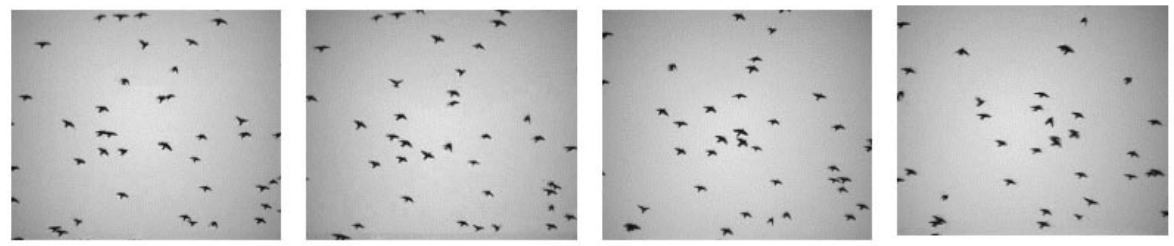

a) Observed sequence of flying birds.
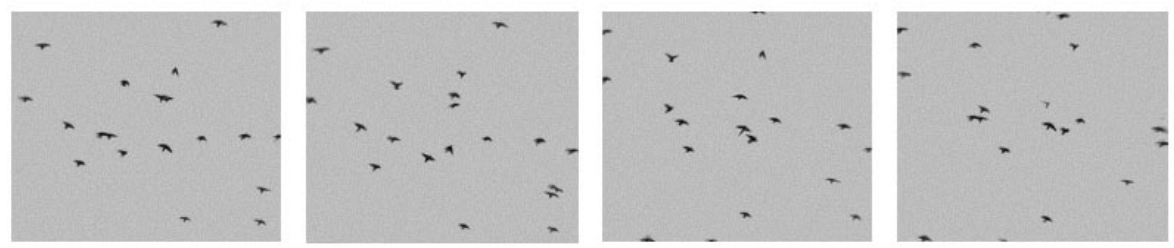

b) Synthesized sequence of flying birds by random sampling (reduced number of birds for photo editing).

Fig. 7. Example of the bird sequence (see bird_obs.avi, bird_syn.avi).

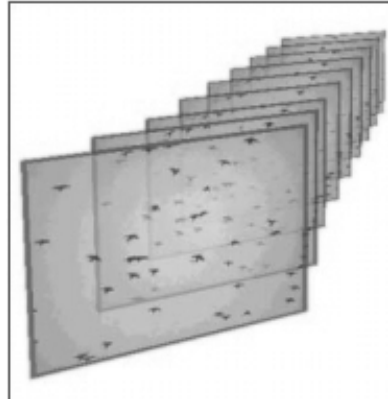

a). input sequence

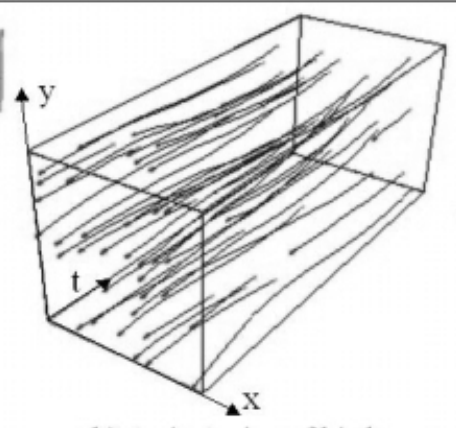

b). trajectories of birds

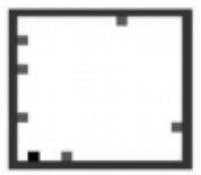

c). birth map (source)

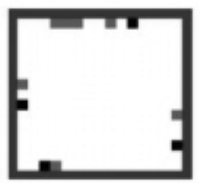

d). death map (sink)

Fig. 8. The computed trajectories of flying birds and the source and sink maps.

\section{Experiments}

We report the results on four textured motion sequences.

1. The snowing sequence. Fig. 2 shows the reconstruction of the snowing images by bases, and a synthesized sequence. For movie, see the observed and synthesized sequence at the attached avi files snow_obs.avi and snow_syn.avi respectively. The algorithm also computes the movetons (snow flake) templates, and random samples are shown in Fig. 3 The trajectories and source/sink maps are shown in Fig 4.

2. The flying bird sequence. Fig. 7.a and b show the observed and synthesize sequences. The animation can be seen at the attached avi files bird_obs.avi and bird_syn.avi. The trajectories and source/sink maps are shown in Fig.8. The 


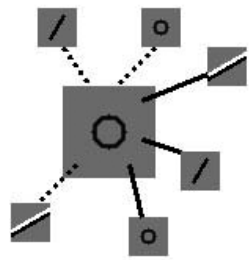

a). deformable template
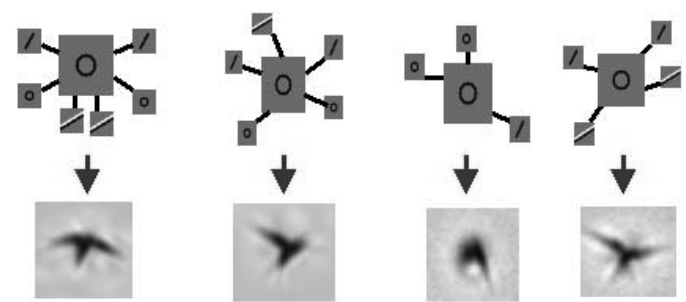

b). instances example: sketch and image
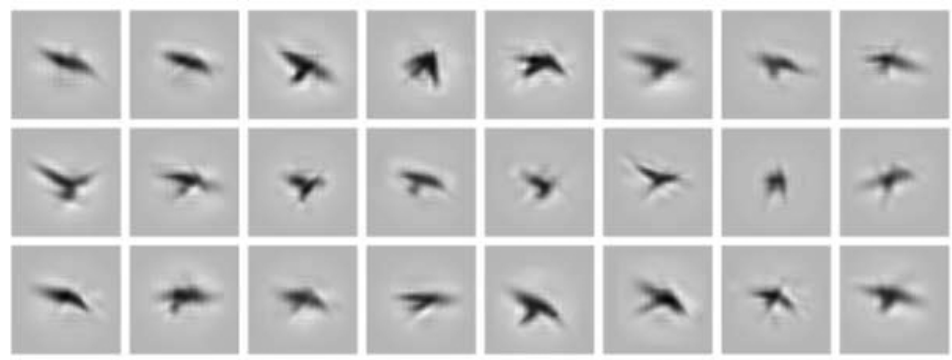

c). more instances sampled from bird templates

Fig. 9. The computed motion elements: flying birds and random examples

birds enter and exit the picture from the image boundary. The maps are rather sparse because we reduced the number of cables (birds) for photo editing effect. Fig.9 shows the deformable templates (a) where a core base is surrounded by a number of small coil bases. The dashed connection means the coil base may or may not appear all the time. A variety of templates and image instances of birds (movetons) are shown in (b) and (c).

3. The firework sequence. Fig. 10.a and b show the observed and synthesized sequences. See attached firework_obs.avi and firework_syn.avi for the movies. The trajectories and source/sink maps are shown in Fig.11. In the synthesis, we edit the birth map $P_{B}(\pi, \lambda)$ by changing its birth rate, assume a uniform distribution for the sources over then lattice. Thus the synthesis has more fireworks.

4. The waterfall sequence. Fig.12 shows the observed and synthesized sequences. See attached waterfall_obs.avi and waterfall_syn.avi for the movies. The trajectories and source sinks are shown in Fig.13. Fig.14 shows 10 typical water drops in the waterfall which are a cluster of bases.

\section{Discussion and Future Work}

The generative model in this paper is motivated by the graphics and vision criteria discussed in Section (1). It learns realistic motion patterns from real data, separates the motion dynamics with photometric and geometric styles, and thus achieves good controllability. For example, we can change the source/sink, 

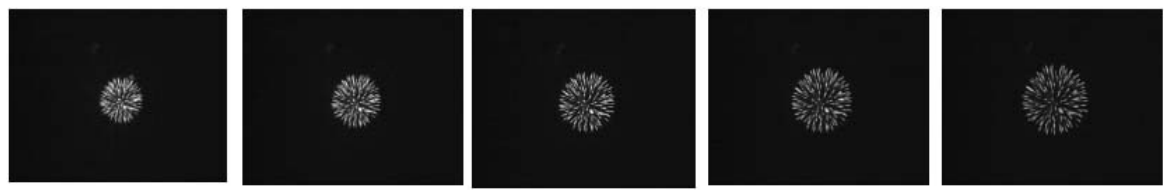

a). observed sequence
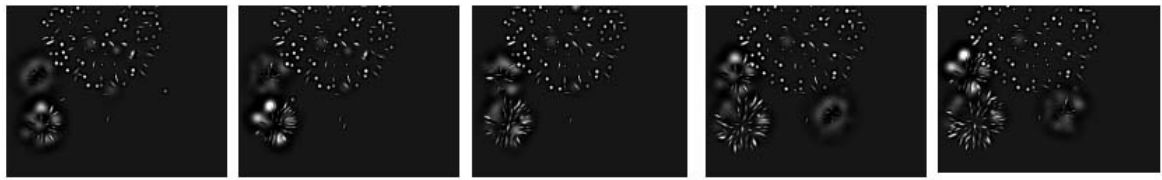

b). synthesized sequence after modifying the birth rate

Fig. 10. Example of the firework sequence (see firework_obs.avi, firework_syn.avi)

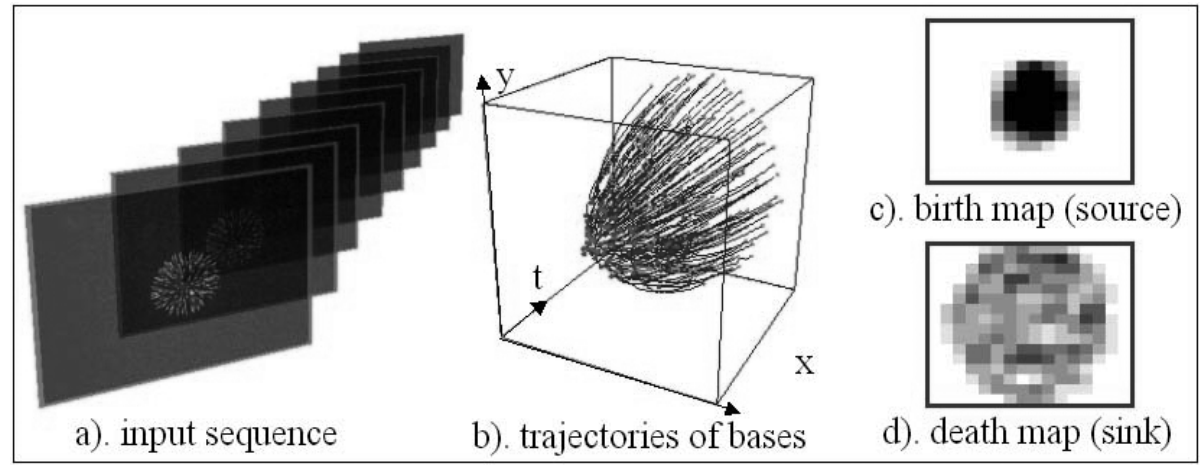

Fig. 11. The computed trajectories of fireworks and the source and sink maps.
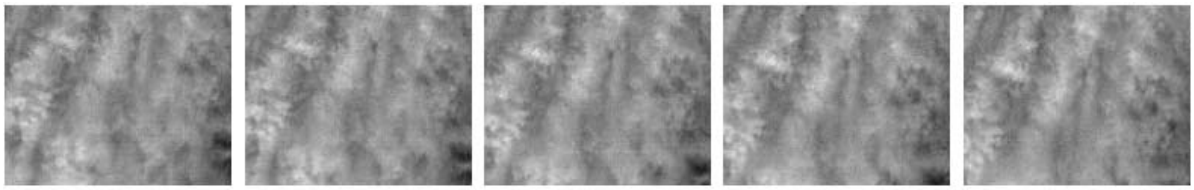

a). observed sequence
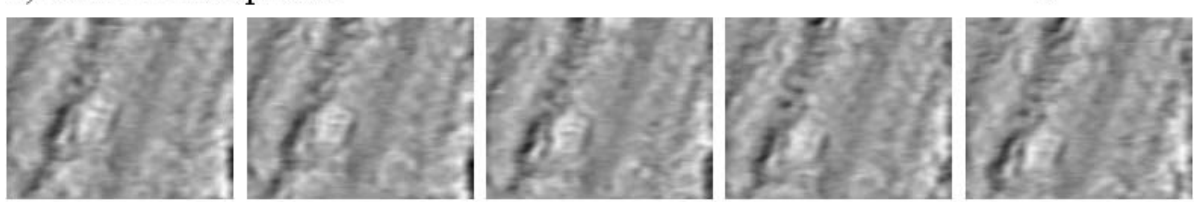

b). synthesized sequence

Fig. 12. Example of the waterfall sequence (see waterfall_obs.avi, waterfall_syn.avi)

alter the dynamics or geometry of movetons by group or by individuals. The representation is semantically meaningful for vision applications as well because 


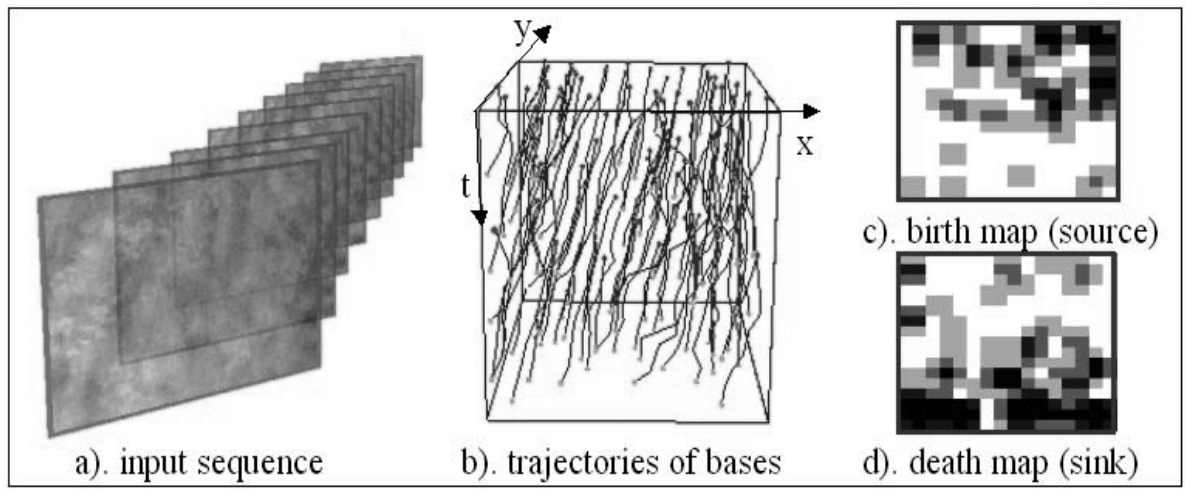

Fig. 13. The computed trajectories of waterfalls and the source and sink maps.
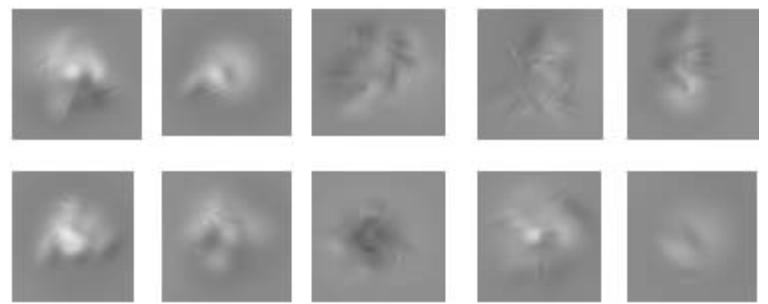

Fig. 14. The random examples of water drops.

the recovered trajectories etc. Needless to say that the generative description $W[0, \tau]$ achieves tremendous compression (usually $10^{2}$-fold) compared to image $\mathbf{I}[0, \tau]$.

In future work we should extend the model in the following aspects.

1. We shall study the spatial interactions of the moving elements, bifurcation and merging of trajectories, and thus integrate good properties of the STAR model to account for lighting variation in motions such as water.

2. We shall study the 3D positions of the moving elements (structure from motion).

\section{Acknowledgments}

We'd like to thank for the support of three research grants, NSF IIS-98-77-127, NSF IIS-00-92-664 and an ONR grant N000140-110-535. Cheng-en Guo and Adrian Barbu are very helpful in programming and creating some of the figures. We also thank the MIT motion texture database. 


\section{References}

1. Z. Bar-Joseph, R. El-Yaniv, D. Lischinski, and M. Werman. "Texture mixing and texture movie synthesis using statistical learning", IEEE Trans on Visualization and Computer Graphics, (to appear).

2. M. J. Black and A. Jepson, "Estimating optical flow in segmented images using variable-order parametric models with local deformations", IEEE Trans on PAMI, Vol. 18, No. 10, pp. 972-986, 1996.

3. S. Chen, D. L. Donoho, and M. A. Saunders, "Atomic decompostion by basis pursuit", Technical preprint, Dept. of Stat., Stanford Univ., 1996.

4. A. D. Cliff and J.K. Ord, "Space-time modeling with an application to regional forecasting", Trans. Inst. British Geographers, 66:119-128, 1975.

5. D. S. Ebert and R. E. Parent, "Rendering and animation of gaseous phenomena by combining fast volume and scaleline A-buffer techniques", SIGGRAPH, 1990.

6. M. G. Gu, "A stochastic approximation algorithm with MCMC method for incomplete data estimation problems", Preprint, Dept. of Stat., McGill Univ. 1998.

7. D. Heeger and J. Bergen, "Pyramid-based texture analysis and synthesis", Proc. of SIGGRAPH, 1995.

8. M. Isard and A. Blake, "Contour tracking by stochastic propagation of conditional density", $E C C V, 1996$.

9. S. Mallat and Z. Zhang, "Matching pursuit in a timef-requency dictionary", IEEE trans. on Signal Processing, vol.41, pp3397-3415, 1993.

10. Y. Meyer, Wavelets: Algorithm and Applications, SIAM, Philadelphia, 1993.

11. B. A. Olshausen and D. J. Field, "Sparse coding with an overcomplete basis set: A strategy employed by V1?", Vision Research, Vo.37, No. 23, pp3311-3325, 1997.

12. W. T. Reeves and R. Blau, "Approximate and probabilistic algorithms for shading and rendering structured particle systems", Proc. of SIGGRAPH, 1985.

13. P. Saisan, G. Doretto, Y.N. Wu, S. Soatto, "Dynamic Texture Recognition," CVPR, 2001.

14. A. Schodl, R. Szeliski, D. Salesin, and I. Essa, "Video texture", Proc. of SIGGRAPH, 2000.

15. S. Soatto, G. Doretto, and Y.N. Wu, "Dynamic texture", ICCV, 2001.

16. A. W. Fitzgibbon, "Stochastic rigidity: image registration for nowhere-static scenes.", Proc. ICCV, pages 662-669, July 2001.

17. M. O. Szummer and R. W. Picard, "Temporal texture modeling", Proc. of Int'l Conf. on Image Processing, Lausanne, Switzerland, 1996.

18. L.Y. Wei and M. Levoy, "Fast texture synthesis using tree structured vector quantization", Proc. of SIGGRAPH, 2000.

19. S. C. Zhu, Y.N. Wu, and D. B. Mumford, "Minimax entropy principle and Its Applications to Texture Modeling", Neural Computation, Vol. 9, Nov. 1997 